\title{
Norms, organizations, and semantics
}

\author{
OLIVIER B OISSIER ${ }^{1}$, MARCO COLOMBET TI ${ }^{2,3}$, \\ M ICHAEL LUCK ${ }^{4}$, JOHN-JULES MEYER ${ }^{5}$ and AXEL POLLERES \\ ${ }^{1}$ Institut Henri Fayol/LSTI, ENS Mines Saint-Etienne, 158 Cours Fauriel, 42023 Saint-Etienne France; \\ e-mail: Olivier.Boissier@emse.fr; \\ ${ }^{2}$ Faculty of Communication Sciences, University of Lugano, via Giuseppe Buffi 13, 6904 Lugano, Switzerland; \\ ${ }^{3}$ Department of Electronics and Information, Politecnico di Milano, piazza Leonardo da Vinci 32, 20133 Milano, Italy; \\ e-mail: marco.colombetti@usi.ch; \\ ${ }^{4}$ Department of Informatics, King's College London, Strand, London WC2R 2LS, United Kingdom; \\ e-mail: michael.luck@kcl.ac.uk; \\ ${ }^{5}$ Department of Information and Computing Sciences, Universiteit Utrecht, Buys Ballot Laboratorium, Princetonplein 5 , \\ 3508 CC Utrecht, P.O. Box 80.089, 3508 TB Utrecht, The Netherlands; \\ e-mail: J.J.C.Meyer@uu.nl; \\ ${ }^{6}$ Siemens AG Österreich, Siemensstrasse 90, 1210 Vienna, Austria; \\ e-mail:axel.polleres@siemens.com
}

\begin{abstract}
This paper integrates the responses to a set of questions from a distinguished set of panelists involved in a discussion at the Agreement Technologies workshop in Cyprus in December 2009. The panel was concerned with the relationship between the research areas of semantics, norms, and organizations, and the ways in which each may contribute to the development of the others in support of next generation agreement technologies.
\end{abstract}

\section{Introduction}

A multi-agent system can be structured into three levels: organizational, coordination, and environment. Semantic concerns cross these levels.

Organizational level: At the organizational level, norms and organizations are closely bound to each other, building what we call normative organizations. Here, the organization specification defines the structure and architecture of the multi-agent system in terms of groups, links, roles, cooperation patterns between actors (users, agents) (such as goaldecomposition schemes, interaction schemes, etc.), resources, and ontologies. Norms define the governance and control for, and within, an organization, and can be considered as regulations constraining agent behaviour, or expectations for behaviour. Such norms can be constitutive, regulative (comprising obligations, prohibitions, and permissions), or technical (i.e., suggestions) norms, and can be defined by an authority or by the agents themselves. The normative state brings information on the violations, fulfilment, hints on when and where to adapt the organization; there are two flows of control between organizational level and the coordination level, leading to two views: a bottom-up or emergent flow; or a top-down or normative flow of processing.

Coordination level: At the coordination level, entities (such as agents) interact with each other. These entities can be agents or organizations themselves, and the interactions between entities can be governed or ungoverned. They take place in the social context of the organizations in which the entities participate. In this context, agreements or contracts bind these entities to the organizational level, as well as to other entities. 
Environment level: Finally, the environment level is the place in which it is possible to situate services, resources, and entities, and must contain support for such processes as service description and discovery of resources.

In this context, this paper integrates the responses to a set of questions from a distinguished set of panelists involved in a discussion at the Agreement Technologies workshop in Cyprus in December 2009. The panel was concerned with the relationship between the research areas of semantics, norms, and organizations, and the ways in which each may contribute to the development of the others in support of next generation agreement technologies.

\section{Norms, policies, and the Semantic Web trust layer}

What is the relation between norms and policies, and what are the similarities and the differences in the way they are designed, negotiated, enforced and maintained? What implications are there for organizations depending on these different perspectives, and to what extent do these address questions relating to the trust layer in the Semantic Web layer cake?

In contemporary distributed systems that are open and dynamic, such as those using Grid technologies, multiple organizations independently provide and use each others' resources. Such systems are now widely used by large scale scientific projects and, increasingly, by businesses wishing to buy computational power and online services as and when needed. Since the aim is that there is typically no centralized control in such systems, the fair and productive distribution of resources, for example, requires distributed automatic mechanisms to resolve conflicting demands, possibly through the use of constraints, policies, or norms.

\subsection{Policies}

The concept of policy itself has multiple interpretations. As stated by Kagal et al. (2003), policies guide the behaviour of entities within a particular domain, such as security, management, and network routing. In this respect, a policy is a specification that describes state and acceptable behaviours. It guides the way in which entities act within a bounded domain by providing rules to constrain their behaviour. The development and use of policies has in turn led to proposals for policy languages and policy management systems. For instance, the policy language Rei (Kagal et al., 2003) is based on deontic concepts and includes primitives for rights, prohibitions, obligations, and dispensations (deferred obligations). The language allows the expression of different kinds of policies (security, privacy, management, conversation, and so on).

As far as they are subject to standards in the World Wide Web Consortium (W3C), policies are typically considered as rules and constraints that model intended behaviours. Within W3C, the Policy Languages Interest Group (PLING) ${ }^{1}$ is the forum for coordinating efforts around policy languages, frameworks, and use cases for policies. Relevant standards range from standard protocols to exchange policies, for example, from P3P (Cranor et al., 2006) to concrete rules languages that should, eventually, allow the description and exchange of such policies as the Rule Interchange Format (RIF) (see the RIF Use Cases and Requirements document (Paschke et al., 2008, Section 10) for a concrete example). Apart from W3C's activities, perhaps the most prominent and established industry standard for describing and exchanging policies is the OASIS eXtensible Access Control Markup Language (XACML; Moses, 2005).

\subsection{Norms}

While policies themselves are typically something individual (my privacy policies in a social network, mail filtering policies, and so on), norms, in contrast, can be regarded as what may be called agreed policies in a community. In this view, policies on the (Semantic) Web build the

1 http://www.w3 .org/Policy/pling/ 
foundation for privacy of personal or organizational data, whereas norms are more important in terms of establishing best practices (for example, how to publish data, and so on).

The term norm is typically considered to derive from philosophy and law, and has a large set of meanings (see Lopez y Lopez et al., 2006; Verhagen, 2007). From this starting point, and as suggested in the introduction, norms have been imported into multi-agent systems mainly to deal with the design of coordination in such systems, for example in relation to seeking to regulate interaction in electronic institutions, electronic commerce systems, or multi-agent organizations. In that sense, norms and policies seem to address similar concerns, but derive from different roots.

However, some argue that in many cases, norms cannot be reduced to policies. For example, Boella and van der Torre (2004) distinguish between regulative norms that describe obligations, prohibitions and permissions, and constitutive norms that regulate the creation of institutional facts like property, marriage and money, as well as the modification of normative system itself. Here, constitutive norms specify what is required in order to be considered part of a system. Conversely, and as stated by Andrighetto et al. (2007), norms are more general due to the social philosophical tradition (Lewis, 1969) that considers norms as conventions emerging in a population (so that they are behaviour patterns without any explicit agreement among agents, emerging from their individual interests). This view is complementary to the legal view proposed in the context of the philosophy of law. The question is how the authoritative perspective promoted by the legal view can be integrated with the individual and emergent perspective promoted by the social philosophy view.

Harmon et al. (2008) distinguish between two kinds of policies: law policies and guidance policies, where the former need to be followed and the latter need not. According to him, the aim of policies is to constrain the system and to add predictability, and in this sense, law policies are very close to norms. Similarly, Bradshaw uses policies in the context of conversations of agents in a way that is somewhat synonymous with that of norms. Interestingly, one can view the distinction between norms and policies by considering that the former relate to groups of societies of agents, while policies are more targeted at individual agents.

More generally, in this global framework, organizations may provide a way to incorporate different explicit policies or norms, providing a social context for their interpretation, or providing an organization management system to ensure their supervision, enforcement, or regimentation. In a complementary approach, policies and norms can express expected behaviours with respect to the management of the organization: access control policies for entering and exiting the organization, organization change policies, and so on.

\subsection{Adoption of norms and policies}

Importantly, one consequence of these divergent origins of the terms policy and norm, and the divergent perspectives on the concepts, is that they are used inconsistently in different scientific communities. For example, what is called a policy in the area of information systems security appears to be very close to what is called a norm in the area of multi-agent systems. This may be a problem in that it is likely to hinder communication between related areas, but it is very difficult to persuade different communities to harmonize their terminologies.

Despite these problems, there may yet be value in these different perspectives. In the area of agreement technologies, for example, we may want to use both terms, with different meanings. While both policies and norms appear to be collections of declaratively represented rules of behaviour, there are some further distinctions that can be identified.

- First, norms specify deontic states (such as obligations, prohibitions, permissions, and the like), and are external in the sense that such deontic states are imposed on agents by some external authority (for example, by an electronic institution or by an authorized individual in such an institution);

- Second, though they can be deontic (as suggested by Rei (Kagal et al., 2003), mentioned above), policies are not necessarily so, since they are internal in the sense that they are individually adopted by an agent without being imposed from the outside. 
For example, an agent's personal policy may be to delete all documentation concerning a business transaction after 2 years, and an external norm (a legal requirement, for instance) may impose the constraint that such documentation should be kept for at least 1 year. In such a case, the agents personal policy would comply with the norm (i.e., if the agents actual behaviour follows the policy, then the norm will be fulfilled).

Why should we care about this distinction? Well, an agent may be programmed in a procedural language to follow a well defined policy, without possessing a declarative representation of it. In such a case, however, the agent would be unable to check if its future behaviour will comply with the norms of an electronic institution. In contrast, a declarative representation, together with a suitable reasoning system, would allow the agent to establish whether this is the case. This matter is clearly related to the issue of trust. We may envision policy-carrying agents that can make their policies public when required. It would then be possible for an agent to check whether its business partners will comply with certain norms.

\subsection{Formalization}

Formalization of both (private and organizational) policies and (community) norms would be useful for various applications (be it Web applications or federated applications across enterprises), such as checking compliance or conformance, alignment of policies, or checking internal consistency or redundancies of policies made explicit. However, formal languages are not yet being used to describe norms and best practices in standardization bodies themselves. Normative documents such as the W3C patent policy (Weitzner, 2004), or best practices documents such as the ones for publishing RDF vocabularies (Berrueta and Phipps, 2008), the Web content accessibility guidelines (Caldwell et al., 2008), or conformance clauses in most standard documents are generally only formulated in natural language.

\subsection{The Semantic Web trust layer}

As to how far questions on policies and norms relate to the trust layer in the Semantic Web layer cake, policy languages are indeed to play a major role here, although many questions (how to enable provenance, signatures, and so on) around this trust layer largely are discussed at a lower level than modelling actual policies. It can probably be expected that the single trust layer as it exists in the various incarnations of the infamous Semantic Web layer cake (see Figure 1) will end up being split into different building blocks and standards, just as was done for the ontology and rules layers; as these layers became populated, they have split up into different interplaying standards that are no longer strictly layered in the sense originally envisaged (e.g., RIF, OWL, RDFS, and SPARQL). However, at this point, the standardization process in the W3C is still two
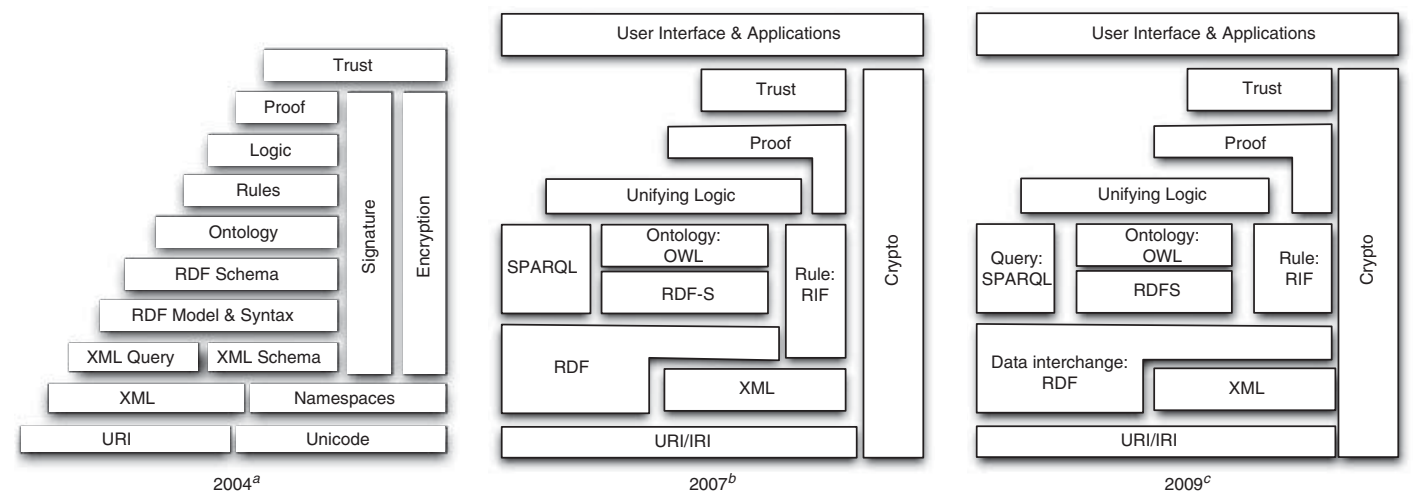

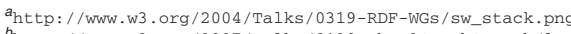

http://wWw.w3.org/2007/Talks/0130-sb-W3CTechSemWeb/layerCake-4.png

Figure 1 Development of the Semantic Web layer cake 
layers down, some way before the trust layer needs to be tackled, and we can expect the simplifying layer picture to change even more drastically over the next few years.

\section{Evolution of norms and organizational change}

Are the evolution of norms and organizational change just two sides of the same coin? What are the factors that trigger these processes and how are they sustained? Is there a consequence of such change for static semantic representations? Conversely, do things like ontology alignment drive organizational change or evolution of norms?

\subsection{Organizational change}

Evolution and change in a multi-agent system can relate to multiple different aspects, including those concerned with the organization, the norms governing the system, or the the semantics. They can be: endogenous (caused by the agents who themselves are members of the organization), or exogenous (caused by observers outside the organization). In this context, the evolution of norms is just one aspect of organizational change, even if it is an important one. Thus, like change itself, norms can be differentiated into: those norms that are part of the external context in which an organization carries out its activities, and provide both constraints and opportunities; and those internal norms that are constitutive of an organization. Both types of norms may evolve, but for different reasons and with different mechanisms. It would be nice to be able to prove, as a matter of organization validation, that acting according to certain internal norms will allow an organization to achieve its goals through actions that comply with the external norms. When this is not the case, a process of change may be triggered (either internal organizational change, or pressure for the change of external norms). Either way, all of this process of evolution and change is sustained by the fact that the systems concerned are open and large scale. The evolution of norms and changes in organizations are strongly related, yet addressing two sides of the same coin: global control of the multi-agent system.

Changes in terms of organization may consist of architectural changes that may impact on the structure of governance that is 'articulated' by the norms. Such changes to the architecture depend on the way in which the organization is characterized or expressed (Hübner et al., 2004). Organizational changes may be in terms of the specification of the architecture (which roles are needed, how to specify groups, how roles are linked together, what cooperation patterns may be specified, etc.) or in terms of the agentification of the architecture (which roles an agent plays, which groups to create, etc.). These organizational changes can be triggered by many different aspects, including: the entry or exit of an agent to the organization; the creation or removal of groups; changes to topology or to the relations between agents, causing new patterns of cooperation; changes to the allocation of roles to agents, or changes in the environment; and, simply, the inefficiency of the architecture (depending on the organization plasticity).

Changes in terms of norms impact on the structure of governance, and can be triggered by: conflicts between norms, the frequency of norm violation or fulfilment; the validity of norms within the organization; and the usefulness of particular norms for the organization, given the agents currently present in the organization. The normative state brings information on the violations, fulfilment, hints on when and where to adapt the organization.

\subsection{Ontology alignment}

Is ontology alignment relevant to all this? It largely depends on how one understands the role of ontologies. Are norms part of certain special ontologies? Or do ontologies just provide the conceptual basis for representing norms? In many cases, the evolution of norms or policies and organizational change mainly relates to merging and aligning existing policies and norms. This issue becomes increasingly important in scenarios, such as big enterprise mergers, where automated support for the alignment of norms and policies would be a real cost-saver. Here, the focus is on the ontology dimension attached to the semantics. If we assume that there may be norms that 
explicitly represent obligations, permissions, or prohibitions in a fashion that relates to the use of ontologies in the system, then changes to norms lead to changes to the system ontology.

It is thus probably appropriate to ask the latter question the other way around as well: whether research in ontology matching, alignment, and merging (for an overview, see for instance Euzenat \& Shvaiko, 2007) can possibly contribute to gaining more insight into how to deal with the alignment of policies and norms. What should be stressed here is that ontology alignment should be viewed broadly. Importantly, description logic-based ontology languages are likely not sufficiently expressive to capture both semantic models and policies, but rule languages and other formalisms are necessary. Unifying semantics of description logics and rules has been a widely discussed topic in the literature over the past few years (see Eiter et al., 2006, 2008 for an overview).

\section{Semantic Web languages and norm-based languages}

How do Semantic Web languages (or standards) and norm-based or organization-based programming languages (or methodologies) relate to each other? Where do you see a potential for crossfertilization?

Clearly, the semantics of organizations and norms need to be expressed somehow, and this relies on ontologies. Indeed, some work has already been done in the area of the Semantic Web and ontologies in relation to social networks, with the Friend of a Friend (FOAF) project (providing a machine-readable ontology describing people and their activities and relations to other people and objects), the Semantically Interlinked Online Communities (SIOC) initiative (which aims to enable the integration of online community information), and so on. In the same way, such ontologies could help with the interoperability of organization models.

While the various languages are concerned with quite different things, they can be used within each other. For example, organization-based programming languages relate to notions like roles, norms, obligations, sanctions, enforcement, regimentation. These may also be included in Semantic Web language ontologies to describe types of organizations as part of the knowledge domains covered by the Semantic Web. Conversely, (parts of) the Semantic Web itself can also be seen as an organization or normative system that may be specified by an organization-based language.

Other connections with Semantic Web technologies consist in the languages that are developed in this domain. Indeed, some have already used Semantic Web languages to express norms (e.g., Lam et al., 2008; Fornara \& Colombetti, 2009); then, using the reasoning capabilities of these languages they are able to detect conflicts of norms (between obligations and permissions, for example). Serious consideration should thus be given to the possibility of adopting W3C (Semantic Web) recommendations as far as possible, in order to achieve interoperability, and to exploit shared ontologies and well-optimized reasoning services. In terms of balancing expressive power and reasoning capacities, an ontology language like OWL (or, more precisely, $O W L 2 D L$ ) is an obvious candidate. Can OWL be used to represent norms? There are three main difficulties: deontic modalities, time, and reasoning under the closed world assumption (CWA), as follows:

Deontic modalities: Norms and the like have a mode (such as obligation, prohibition, and permission) and a content (what is obligatory, forbidden, or permitted), and agents should be able to reason about both. As far as reasoning about content is concerned (e.g, inferring that a bank transfer fulfils an obligation to pay), OWL seems to be the right choice. However, we also want our agents to reason about modalities, and this is difficult (maybe even impossible) to do in OWL. But seriously consideration should be given to the option of building deontic reasoners on top of OWL reasoners, instead of trying to build stand-alone deontic reasoners from scratch.

Time: Temporal reasoning (e.g., of the CTL type) is a necessary component of deontic reasoning, because obligations and the like occur in time, and are subject to deadlines. It is not clear that the problem can be solved by providing a suitable upper ontology of time in OWL. The language is just not powerful enough for this purpose; for example, the OWL 
Time Ontology ${ }^{2}$ has all the basic concepts of Allens interval algebra, but is not able to represent most of the relevant axioms, which makes it unfit for temporal reasoning. Therefore, a temporal extension of OWL would be very valuable.

Reasoning under the CWA: Closed world reasoning is essential in most interactions. For example, the fact that an agent is unable to prove that it has paid an invoice is typically sufficient to assume that the payment has not been made. Even if standard OWL reasoning assumes an open world, closed world reasoning can already be effectively simulated by exploiting SPARQL queries, with some post-processing. However, a more rigorous approach (e.g., based on the epistemic operator K) would be preferable.

There is still a huge potential in terms of making the existing Semantic Web standards themselves interplay better, and this is where more research is still required in order to solve several problems, before the trust layer of the Semantic Web can be populated with standards. While protocols and languages like $\mathrm{P} 3 \mathrm{P}$ and XACML are emerging, it will become important to establish ways in which to tie these with domain ontologies in RDFS and OWL, or to embed rule-based descriptions of policies (e.g., given in RIF) in formal descriptions of policies and norms. Still, what is important to observe is that all these languages and standards are already being used by communities of increasing sizes. The main question is thus not how to promote or establish new languages and standards to increase the existing language zoo, but how to close the gaps between the existing standards, or how to build frameworks that make them interplay smoothly. Wherever research in norm-based or organizationbased programming languages can contribute here, there is a good chance for practical impact.

\section{Standards}

The Semantic Web is heavily driven by the development and adoption of standards. How should these impact on practical developments in the areas of norms and organizations? What scope is there for the development and adoption of similar standards for organizations and norms?

In a narrow view, one could claim that the Semantic Web is actually all about standardization since it is an activity that emerged within the World Wide Web Consortium - a standardization body. There are important lessons to be learned from these standardization efforts in the Semantic Web area: the bottom-up population of an architectural idea (symbolized by the Semantic Web layer cake in Figure 1) by standards is beginning to being picked up on a wide scale; RDF is becoming increasingly popular on the web, and lightweight ontologies such as $\mathrm{FOAF}^{3}$ or SIOC (Bojārs et al., 2007) are being used to publish more and more structured content on the web following the so called linked data principles (Berners-Lee, 2006), making a big fraction of the web machine-understandable. Yet those ontologies being widely used on the emerging web of data (Polleres \& Huynh, 2009) are not necessarily complex. For instance, SIOC, an ontology for describing online communities and their conversations consists only of a handful of concepts and relations, but most of the effort of the SIOC initiative went into finding agreement on these common terms and promoting the ontology's usage on the web, for example by developing exporters and tools (see $h t t p: / / w w w . s i o c-p r o j e c t . o r g /$ ). It is such efforts that enable practical deployment of Semantic Web technologies.

Thus, the lesson learnt is that standards and technologies make only as much sense as the level of their eventual deployment, following Metcalfe's law that the value of telecommunications technologies - and this law seems to apply even more to web technologies - increases proportionally with the the square of its users. Technologies that help to enforce policies or establish norms on the web will need to follow the same principles.

In the domain of multi-agent organizations, there are many different models, with different expressiveness and different dimensions (see, e.g., Boella et al., 2005; Coutinho et al., 2009b). This large

2 http://www.w3.org/TR/owl-time/

3 http://xmlns.com/foaf/spec/ 
number of models is certainly due to the fact that the a particular organization structure determines the multi-agent system architecture and the nature of its patterns of cooperation, so that all of the dimensions of such systems become relevant. Clearly, semantic interoperability between all these models is important (Coutinho et al., 2009a) and, organizations themselves do have a certain standard ontology, containing such elements as roles, and leading to the emergence of at least interesting possibilities. While the Semantic Web and agent organizations are distinctly not the same category of thing, and it's not clear that we can, or even should, transfer the methodology of the Semantic Web directly to agent organizations, it seems reasonable to consider the relationship of the two paradigms.

The success of the agreement technologies, both technically and culturally, will crucially depend on the adoption of standards: we cannot even conceive of an open interaction system without shared standards! Premature attempts to specify standards, however, can be misleading (as some claim was the case with FIPA's agent communication language, for example).

\section{Norms and context}

Should one aspire to make social norms, such as 'belligerence is not nice' or 'be polite', become part of the context where agreements take place? For example, in the Semantic Web, it is implicit that groups or organizations adopt a norm on which vocabulary or ontology to use. Should these norms be explicitly represented? What about norms that specify use of these across multiple groups or organizations? How might this be done and what are the technical challenges raised by doing so?

Many human social norms (like 'be polite') concern aspects of interaction that are not relevant for artificial agents. But other type norms (like 'be pertinent', concerning communicative exchanges) seem to be relevant and important. More precisely, the pragmatics of communication (which is concerned with such norms) is an interesting area of research for agent communication in general, and for agreement technologies in particular. To be of any use, a norm must concern aspects of behaviour that can be effectively monitored at run time. But it may be hard to judge, for example, whether a counter-proposal is reasonable (in the sense that, e.g., if A proposes to B that it will provide a service for $€ 1000$, it will be unreasonable for $B$ to counter-propose that the same service be provided for $€ 1$ ). To establish what kinds of useful social norms can be effectively represented is a difficult open problem.

Norms can be used to regulate open multi-agent systems in which (heterogeneous) agents may join and leave at any time. Since such a normative multi-agent system determines a kind of agreement or contract by imposing conditions under which these agents may join, it is important that these are explicitly represented (e.g., in a declarative form, such as expression in a deontic logic or derivatives thereof, such as counts-as rules). Then, verification techniques, for instance, may become applicable, which may of course be very useful (depending on the application).

More specifically, multi-agent technologies are suited to addressing application domains in which key challenges are openness, scalability, evolution, and decentralization. In this context, norms that define the structure of governance of the system must be represented. In particular, such a clear and explicit representation is necessary if we want agents to reason about the norms and potentially bring about changes to them, or cause their evolution over time. Thus, for instance, in relation to the specific question concerning ontologies, if we want agents to change the ontology they use in a specific organization, we need norms that specify which ontology to use. However, there are many challenges raised by expressing norms across multiple groups or organizations. For example, how should such norms be monitored and enforced, and by which authority or organization management system? What is the minimal ontology required by agents in order to understand and reason about the different organizations and norms: should agents possess an ontology about their organization model and their norm model; might they have different ontologies for such models; and is it possible to have a standard ontology for organizations?

In the context of the web, best practices and norms are indeed largely not (yet) being made explicit, and indeed it is questionable whether doing so would have measurable benefits. Many de facto standards on the web did not emerge from standardization bodies at all, but rather 
from grass roots efforts, yet standardization bodies can still help by rubber-stamping agreed technologies to make them usable beyond specialized communities. In fact, standardization processes like those within $\mathrm{W} 3 \mathrm{C}$, with mechanisms such as member submissions for proposing technologies that have been proven useful in practice for standardization, try to encompass such movements.

\section{Future prospects}

In your view, what is the greatest contribution that the areas of semantics, norms and organizations can make to the others? What are you doing to make this happen? What do you recommend to others to do?

The greatest contribution is perhaps the integration and cross-fertilization of the different research undertaken in each of the three areas of semantics, norms, and organizations. However, there is a sense in which the areas of semantics, norms, and organizations have been conceived as a stack. Indeed, organizations require norms, and norms require ontologies. The interaction between these areas can be neither purely top-down (organizations providing requirements for norms, and norms doing the same for semantics), nor purely bottom-up (semantics offering concepts and technologies for norms, which in turn offers concepts and technologies to organizations). Clearly, a more circular type of interaction is needed and, in practical terms, each community should be extremely sensitive to what is going on in the other two, in terms of both requirements and offers.

Among the specific areas that can be identified for future work are the following:

- deontic, temporal, and closed world reasoning using ontologies;

- representation of organizations as deontic entities;

- ontologies of norms (as part of a knowledge domain);

- norms on (the use of) ontologies (for instance in ontology alignment);

- semantics of organizational models;

- norms and alignment of different organizations, permitting an agent to move from one organization to another, and executing within each organization subject to the different norms that exist within.

\section{References}

Andrighetto, G., Conte, R., Turrini, P. \& Paolucci, M. 2007. Emergence in the loop: simulating the two way dynamics of norm innovation. In Normative Multi-agent Systems, Boella, G., van der Torre, L. \& Verhagen, H. (eds). Dagstuhl Seminar Proceedings, 07122. Internationales Begegnungs- und Forschungszentrum für Informatik (IBFI).

Berners-Lee, T. 2006. Linked Data - Design Issues. http://www.w3 .org/DesignIssues/LinkedData.html.

Berrueta, D. \& Phipps, J. (eds) 2008. Best Practice Recipes for Publishing RDF Vocabularies. W3C Working Group Note. http://www.w3.org/TR/swbp-vocab-pub/.

Boella, G. \& van der Torre, L. 2004. Regulative and constitutive norms in normative multiagent systems. In Principles of Knowledge Representation and Reasoning: Proceedings of the 9th International Conference (KR2004), Dubois, M. W. D. \& Welty, C. A. (eds). AAAI Press, 255-265.

Boella, G., Odell, J., van der Torre, L. \& Verhagen, H. (eds) 2005. An Interdisciplinary Perspective - AAAI Fall Symposium. Technical report FS-05-08.

Bojārs, U., Breslin, J. G., Berrueta, D., Brickley, D., Decker, S., Fernández, S., Görn, C., Harth, A., Heath, T., Idehen, K., Kjernsmo, K., Miles, A., Passant, A., Polleres, A., Polo, L. \& Sintek, M. 2007. SIOC Core Ontology Specification. W3C member submission. http://www.w3.org/Submission/sioc-spec/.

Caldwell, B., Cooper, M., Reid, L. G. \& Vanderheiden, G. (eds) 2008. Web Content Accessibility Guidelines (WCAG) 2.0. W3C Recommendation. http://www.w3.org/TR/WCAG20/.

Coutinho, L. R., Brandao, A. A. F., Sichman, J. S. \& Boissier, O. 2009a. Model-driven integration of organizational models. In Agent-Oriented Software Engineering IX, 9th International Workshop, AOSE 2008, Luck, M. \& Gomez-Sanz, J. (eds), Revised Selected Papers, Lecture Notes in Computer Science, 5386, 1-15. Springer.

Coutinho, L. R., Sichman, J. S. \& Boissier, O. 2009b. Modelling dimensions for agent organizations. In Handbook of Research on Multi-Agent Systems: Semantics and Dynamics of Organizational Models, Dignum, V. (ed.). Information Science Reference Publisher, 2-18. 
Cranor, L., Dobbs, B., Egelman, S., Hogben, G., Humphrey, J., Langheinrich, M., Marchiori, M., Presler-Marshall, M., Reagle, J., Schunter, M., Stampley, D. A. \& Wenning, R. 2006. The Platform for Privacy Preferences 1.1 (P3P1.1) Specification. W3C Working Group Note. http://www.w3.org/TR/ P3P11/.

Eiter, T., Ianni, G., Krennwallner, T. \& Polleres, A. 2008. Rules and ontologies for the Semantic Web. In Reasoning Web 2008, Baroglio, C., Bonatti, P. A., Maluszynski, J., Marchiori, M., Polleres, A. \& Schaffert, S. (eds), Lecture Notes in Computer Science, 5224, 1-53. Springer, URL http://www. polleres. net/publications/eite-etal-2008.pdf.

Eiter, T., Ianni, G., Polleres, A., Schindlauer, R. \& Tompits, H. 2006. Reasoning with rules and ontologies. In Reasoning Web 2006, Barahona, P., Bry, F., Franconi, E., Henze, N. \& Sattler, U. (eds), Lecture Notes in Computer Science, 4126, 93-127. Springer, URL http://www.polleres.net/publications/ eit-etal-2006_rowSchool.pdf.

Euzenat, J. \& Shvaiko, P. 2007. Ontology Matching. Springer, ISBN 3-540-49611-4.

Fornara, N. \& Colombetti, M. 2009. Ontology and time evolution of obligations and prohibitions using Semantic Web technology. In Declarative Agent Languages and Technologies VII, 7th International Workshop, DALT 2009, Budapest, Hungary, May 11, 2009, Baldoni, M., Bentahar, J., van Riemsdijk, M. B. \& Lloyd, J. (eds), Revised Selected and Invited Papers, Lecture Notes in Computer Science, 5948, 101-118. Springer.

Harmon, S. J., DeLoach, S. A. \& Robby 2008. Trace-based specification of law and guidance policies for multi-agent systems. In Engineering Societies in the Agents World VIII, 8th International Workshop, ESAW 2007, Athens, Greece, October 22-24, 2007, Artikis, A., O'Hare, G. M. P., Stathis, K. \& Vouros, G. A. (eds), Revised Selected Papers, Lecture Notes in Computer Science, 4995, 333-349. Springer, ISBN 978-3540-87653-3.

Hübner, J. F., Sichman, J. S. \& Boissier, O. 2004. Using the MOISE+ for a cooperative framework of MAS reorganisation. In Advances in Artificial Intelligence: SBIA 2004, 17th Brazilian Symposium on Artificial Intelligence, Bazzan, A. L. C. \& Labidi, S. (eds), Lecture Notes in Computer Science, 3171, 506-515. Springer.

Kagal, L., Finin, T. \& Joshi, A. 2003. A policy language for a pervasive computing environment. In Proceedings of the IEEE 4th International Workshop on Policies for Distributed Systems and Networks, Lake Como, Italy, 63-74.

Lam, J. S.-C., Guerin, F., Vasconcelos, W. \& Norman, T. J. 2008. Representing and reasoning about norm-governed organisations with Semantic Web languages. In Proceedings of the 6th European Workshop on Multi-Agent Systems (EUMAS 2008), Bath, UK.

Lewis, D. 1969. Convention: A Philosophical Study. Harvard University Press.

Lopez y Lopez, F., Luck, M. \& d'Inverno, M. 2006. A normative framework for agent-based systems. Computational Mathematical Organization Theory 120(2-3), 227-250.

Moses, T. (ed.) 2005. eXtensible Access Control Markup Language (XACML) Version 2.0. OASIS Standard.

Paschke, A., Hirtle, D., Ginsberg, A., Patranjan, P.-L. \& McCabe, F. 2008. RIF Use Cases and Requirements. W3C Working Draft. http://www.w3.org/TR/rif-ucr/.

Polleres, A. \& Huynh, D. (eds) 2009. Journal of Web Semantics, Special Issue: The Web of Data, 7.

Verhagen, H. 2007. Normtypologies. In Normative Multi-agent Systems, Boella, G., van der Torre, L. \& Verhagen H. (eds), Dagstuhl Seminar Proceedings, 07122, Dagstuhl, Germany. Internationales Begegnungs- und Forschungszentrum für Informatik (IBFI). URL http://drops.dagstuhl.de/opus/ volltexte/2007/930.

Weitzner, D. J. (ed.) 2004. W3CPatent Policy. http://www.w3.org/Consortium/Patent-Policy/. 